\title{
THE HEIGHT DEPENDENCE OF GRANULAR MOTION
}

\author{
Anastasios Nesis \\ Kiepenheuer Institut fli Sonnenphysik \\ Freiburg i.Br., W. Germany
}

Abstract

Spectrograms of $\mathrm{Mg} \mathrm{I}$-absorptions lines have been registrated at different positions of the darping wings. The corresponding heights within the atmosphere have been approximated by the Eddington-Barbiex approximation. We calculated the coherence between the intensity fluctuations in the continuum and those of the higher layers. We found a rather flat gradient up to a height of $100 \mathrm{~km}$ and above this a steep decrease of coherence. From this result we can conclude that above a height of 100 $\mathrm{km}$ any fluctuations of intensity are not due to ordinary convective processes. However, up to a height of $400 \mathrm{~km}$ we found a coherence between the velocity of granulation and its intensity fluctuations.

NUMERICAL SIMULATIONS OF THE SOLAR GRANULATION

Åke Nordlund

NORDITA, Blegdamsvej 17

Dk-2100, København $\varnothing$, Denmark

Abstract

Numerical simulations of the convective granular motions in the solar photosphere are presented. Realistic background physics allows a detailed comparison with observed characteristics of the solar granulation. The numerical methods are based on a bivariate Fourier representation in the horizontal plane, combined with a cubic spline representation in the vertical direction. Using a numerical grid with $16 \times 16 \times 16$ grid points, which cover a unit cell of dimension $\simeq 3600 \times 3600 \times 1500 \mathrm{~km}$, granular motions have been followed over several turnover times. The simulated motion shows the characteristics of granular motion. The evolution of large granules into bright rings ("Exploding granules") is a consequence of the accumulating excess pressure at the granule center, necessary to support the horizontal velocities required by the contin- 
uity equation. The increasing pressure evenutally inhibits further upward motion at the granule center, which cools radiatively and shows up as a dark center in the expanding granule. 